\title{
A Linear Programming Approach to Cross-Layer Optimization in Multiclass VSG-CDMA Over Rayleigh Fading
}

\author{
Pongsatorn Sedtheetorn, Member, IEEE, and Khairi Ashour Hamdi, Senior Member, IEEE
}

\begin{abstract}
This paper presents a new linear programming approach for throughput maximization on the uplink of a multiclass variable spreading gain code-division multiple-access (CDMA) multicellular system in Rayleigh fading for both binary phase-shift keying (BPSK) and quaternary phase-shift keying (QPSK) modulations. Based on the improved Gaussian approximation technique, we derive exact closed-form expressions of the outage probability, which are used as a physical (PHY) layer constraint of the maximization problem. We show that it is possible to transform the nonlinear constraint into a set of equivalent linear expressions. This facilitates the formulation of a new linear throughput maximization that noticeably requires less computational complexity than the known nonlinear approaches. Furthermore, due to the simplicity of the linear approach, we can include the constraints of such higher layers, i.e., media access control (MAC) and call admission control (CAC) layers, into the proposed formula. Accordingly, we introduce two linear programming optimization formulas. One is for MAC-PHY optimization, and another is for cross-layer optimal CAC policy. In the case of MAC-PHY optimization, the throughput is maximized, at low background noise level, when every user retains the same bit energy, particularly in the case where the difference in data rates is high. Nonetheless, at high levels of background noise, the throughput would be maximized when a larger amount of bit energy is allocated to the high-rate users. For the joint optimal policy, the throughput and the blocking probability are optimized and improved up to $50 \%$ in comparison to those of the conventional CAC policy, which is known as the complete sharing policy.
\end{abstract}

Index Terms-Code division multiaccess, fading channels, interference, land mobile radio cellular systems, linear programming.

\section{INTRODUCTION}

$\mathbf{T}$ HIS paper addresses the cross-layer optimization problem for the uplink throughput of a multiclass variable spreading gain (VSG) code-division multiple-access (CDMA) multicellular system in which the different classes of users are distinguished by their transmission speeds and spreading gains.

Manuscript received May 5, 2006; revised December 19, 2006, May 17, 2007, and August 6, 2007. The review of this paper was coordinated by Prof. J. H. Cho.

P. Sedtheetorn was with the School of Electrical and Electronic Engineering, University of Manchester, M60 1QD Manchester, U.K. He is now with the Department of Electrical and Computer Engineering, Mahidol University, Nakhon Pathom 73170, Thailand (e-mail: egpse@mahidol.ac.th).

K. A. Hamdi is with the School of Electrical and Electronic Engineering, University of Manchester, M60 1QD Manchester, U.K. (e-mail: k.hamdi@manchester.ac.uk).

Digital Object Identifier 10.1109/TVT.2007.909259
Recently, the throughput maximization problem on multiclass CDMAs has gained considerable research interest, where several optimization techniques have been presented, e.g., [1]-[8]. The constraints in most of the previous research are defined in terms of signal-to-interference-plus-noise ratio (SINR) [1]-[3] or outage probability [5], which is derived from the inaccurate simple Gaussian approximation technique [17]. Because the constraints are generally nonlinear, previous optimization techniques are based on either nonlinear programming [1]-[3] or analytical differentiation methods [6]-[8]. Nonetheless, it is well known that nonlinear programming leads to a considerable amount of computational burden, particularly when the number of classes or users is large [5], whereas analytical differentiation techniques are confined to some simplified system models [7]. These drawbacks limit the previous study to small systems with a couple of classes of users. Moreover, due to the complexity of nonlinear programming, it seems impossible for the traditional techniques to deal with crosslayer optimization in which additional quality-of-service (QoS) constraints from higher layers are included.

In contrast to the previous study, we formulate a throughput maximization that can be solved by a linear programming method. We introduce a new set of linear constraints for the physical (PHY) layer, which is accurately computed based on the improved Gaussian approximation (IGA) technique [20]. This facilitates the construction of a maximization problem as a linear programming formula, which is readily solved by wellknown methods, e.g., the simplex method [31]. In addition, the proposed formula is extendable to multiclass systems, as well as cross-layer optimizations, that include some important constraints of the higher layers, i.e., media access control (MAC) and call admission control (CAC) layers.

The general assumptions of this paper are as follows. The system model is analyzed based on the accurate IGA technique [20] in which binary phase-shift keying (BPSK) and quaternary phase-shift keying (QPSK) modulations are taken into account. In the case of multicellular analysis, the cell shape is assumed to be circular. The transmission channel is assumed to have Rayleigh fading with perfect (slow) power control. The MAC layer is represented by an $\mathrm{M} / \mathrm{M} / \mathrm{m} / \mathrm{m}$ queueing model [25], and the admission control is characterized by a semi-Markov decision process (SMDP) [26]-[29].

The remainder of this paper is organized as follows. In Section II, we show the system model for a multiclass VSGCDMA and derive new exact expressions for the outage 
probabilities in the case of BPSK and QPSK. In Section III, we formulate a new linear programming approach for MAC-PHY throughput maximization. In Section IV, a joint optimal admission policy (across CAC, MAC, and PHY layer) is proposed. Numerical results and discussions are given in Section $\mathrm{V}$, and conclusions are drawn in Section VI.

\section{PHY LAYER ANALYSIS}

This section describes the system models of a multiclass VSG-CDMA in the case of BPSK and QPSK modulations. The SINRs are analyzed using the IGA technique from which new exact closed-form expressions of the outage probability are derived. At the end of this section, the analysis is extended to include intercell interferences.

\section{A. BPSK-Modulated System}

Consider a multiclass VSG-CDMA system in an additive white Gaussian noise (AWGN) Rayleigh fading channel. Assume that users' signals are asynchronously transmitted and signature codes are independent and randomly generated. Denote $m \in 1,2, \ldots, M$, which is the normalized rate of class- $m$ traffic (normalized to class- 1 rate, which is considered as the basic rate). Let $x_{m}$ be the number of class- $m$ signals, and the data bit stream of a class- $m$ signal is given by

$$
b_{x, m}(t)=\sum_{j=-\infty}^{\infty} b_{j, x, m} \operatorname{rect}\left(\frac{t}{T_{b} / m}-j\right)
$$

where $x \in\left\{1, \ldots, x_{m}\right\}$ is the index of each signal, $j$ stands for the index of individual data bits, $T_{b}$ is the class- 1 bit transmission time, $b_{j, x, m}$ is a sequence of independent and identically distributed random variables (i.i.d. RVs) that takes values in $\{+1,-1\}$ with equal probability, $\operatorname{rect}(t)$ is a unitamplitude rectangular pulse for $0 \leq t<1$, and $\operatorname{rect}(t)=0$, otherwise. Accordingly, a received class- $m$ signal is

$$
v_{x, m}(t)=h_{x, m} \sqrt{2 s_{m}} b_{x, m}(t) a_{x, m}(t)
$$

where $s_{m}$ represents the local mean power of the signal, and $\left\{h_{x, m}\right\}, x=1, \ldots, x_{m}, m=1, \ldots, M$ are the complex channel gains for slow flat Rayleigh fading. It is assumed that $\left\{h_{x, m}\right\}$ are mutually independent zero-mean complex Gaussian RVs [23] with $E\left[h_{x, m} h_{y, n}^{*}\right]=1$ only if $x=y$ and $m=n$ and is equal to 0 , otherwise.

$a_{x, m}(t)=\sum_{j=-\infty}^{\infty} a_{j, x, m} \psi\left\{\left[t /\left(T_{b} / G\right)\right]-j\right\}$ are the signature sequence signals, where $G$ is the processing gain of the basic-rate signals (class-1 signals), which is equal to the number of chips per bit duration. $T_{b} / G$ is the chip interval, which is identical for all classes. $\psi(t)$ is a chip waveform function, which is assumed to be time limited in $\left[0, T_{b} / G\right]$ and normalized such that $\left[1 /\left(T_{b} / G\right)\right] \int_{0}^{T_{b} / G}|\psi(t)|^{2} d t=1$. The signature sequences $\left\{a_{j, x, m}\right\}$ are modeled as a sequence of i.i.d. RVs with $\operatorname{Pr}\left\{a_{j, x, m}=+1\right\}=\operatorname{Pr}\left\{a_{j, x, m}=-1\right\}=1 / 2$. It is clear to say that each bit of a transmitted signal is spread by multiplying with a newly chosen random signature sequence. This model is widely employed in [13]-[17], [21], and [22].
It is important to state that perfect power control at the base station is assumed in this paper so that the received powers from the same class of traffic are identical. This assumption is commonly used in CDMA system model analysis, e.g., [13]-[22].

Let the receiver be locked onto an arbitrary reference class- $n$ signal (signal number 1) in the presence of other multipleaccess interferences (MAIs). Denote $r(t)$ as the composite signal at the receiver front end. Then, we obtain

$$
r(t)=v_{1, n}(t)+\sum_{m=1}^{M} \sum_{\substack{x=1, m \neq n \\ x=2, m=n}}^{x_{m}} v_{x, m}\left(t-\tau_{x, m}\right)+n(t)
$$

where $\tau_{x, m}$ represents the time offset of the $x$ th class- $m$ signal with respect to the reference. $\eta(t)$ is the low-pass complex value AWGN with a two-sided spectral density $N_{0}$.

Assume a conventional correlator receiver, and let $Z_{j, 1, n}$ be the normalized decision variable of the $j$ th bit of the reference user. At the decision time instance $t=(j+1) T_{b} / n$, the output of the correlator receiver is given by [17]

$$
\begin{aligned}
Z_{j, 1, n}=\operatorname{Re}\{ & \pm\left|h_{1, n}\right| \sqrt{2 s_{n}}+\sum_{m=1}^{M} \sum_{\substack{x=1, m \neq n \\
x=2, m=n}}^{x_{m}} \\
& \left.\times \chi_{m} \sqrt{2 s_{m}} e^{-i \arg \left(h_{1, n}\right)} h_{x, m} \mathfrak{W}_{x, m}+\eta\right\}
\end{aligned}
$$

where $\eta$ is the complex Gaussian RV with variance $N_{0} /\left(T_{b} / n\right)$. $i=\sqrt{-1}$ stands for an imaginary number. $\chi_{m}$ is the class- $m$ activity factor, which is a Bernoulli distributed RV, and

$$
\chi_{m}= \begin{cases}1, & \text { active with probability } q_{m} \\ 0, & \text { otherwise }\end{cases}
$$

$\mathfrak{W}_{x, m}$ is the MAI component in the interval $\left[j T_{b} / n,(j+1)\right.$ $\left.T_{b} / n\right]$ of the reference $j$ th bit due to the interfering $x$ th class- $m$ signal [19], which is given by

$$
\begin{aligned}
\mathfrak{W}_{x, m}=\frac{1}{T_{b} / n} \int_{j T_{b} / n}^{(j+1) T_{b} / n} b_{x, m} & \left(t-\tau_{x, m}\right) \\
& \times a_{x, m}\left(t-\tau_{x, m}\right) a_{1, n}(t) d t .
\end{aligned}
$$

It is shown in [19] and [20] that when $G \gg 1, \mathfrak{W}_{x, m}$ become conditionally independent zero-mean Gaussian RVs (conditioned on the relative time offset), with the conditional variance

$$
\operatorname{Var}\left(\mathfrak{W}_{x, m} \mid\left\{\delta_{x, m}\right\}_{x, m}\right)=\frac{1}{G / n}\left[\rho^{2}\left(\delta_{x, m}\right)+\hat{\rho}^{2}\left(\delta_{x, m}\right)\right]
$$

where $\delta_{x, m} \in[0,1],\left\{x=1,2, \ldots, x_{m}, m=1,2, \ldots, M\right\}$ is the normalized time offset modulo of the chip interval $T_{b} / G \cdot \rho(\cdot)$ and $\hat{\rho}(\cdot)$ are defined as the continuous partial correlation functions of the chip wave form, i.e., $\hat{\rho}(\tau)=$ $1 /\left(T_{b} / G\right) \int_{\tau}^{T_{b} / G} \psi(t) \psi(t-\tau) d t$ and $\rho(\tau)=1-\hat{\rho}(\tau)$. 
Therefore, when we condition on the RVs $\left\{\chi_{m}, h_{x, m}\right.$, $\left.\delta_{x, m}, \quad x=1,2, \ldots, x_{m}, \quad m=1,2, \ldots, M\right\}$, the decision variable becomes a conditionally Gaussian variable having a conditional variance given by

$$
\begin{aligned}
& \operatorname{Var}_{Z_{j, 1, n}}\left[Z_{j, 1, n} \mid\left\{\chi_{m}, h_{x, m}, \delta_{x, m}\right\}_{x, m}\right] \\
& =\frac{1}{G / n} \sum_{m=1}^{M} \sum_{\substack{x=1, m \neq n \\
x=2, m=n}}^{x_{m}} \chi_{m} s_{m}\left(\operatorname{Re}\left\{h_{x, m}\right\}\right)^{2} \\
& \quad \times\left[\rho^{2}\left(\delta_{x, m}\right)+\hat{\rho}^{2}\left(\delta_{x, m}\right)\right]+\frac{n s_{1}}{2\left(E_{1} / N_{0}\right)}
\end{aligned}
$$

where $E_{1}=s_{1} T_{b}$ is the energy per bit interval of class-1 traffic, and we have used the fact that $\chi_{m}^{2}=\chi_{m}$ (which is the property of Bernoulli RVs).

Hence, the SINR of a class- $n$ reference signal is $\operatorname{SINR}_{n}=(1 / 2)\left[E_{Z_{j, 1, n}}^{2}\left[Z_{j, 1, n}\right] / \operatorname{Var}_{Z_{j, 1, n}}\left[Z_{j, 1, n}\right]\right]$, where $E_{Z_{j, 1, n}}^{2}\left[Z_{j, 1, n}\right]=s_{n}\left|h_{1, n}\right|^{2}$.

Let $\zeta_{n}$ be the targeted SINR of class- $n$. The outage probability is expressed in (9), shown at the bottom of the page.

Note that $\left|h_{1, n}\right|^{2}$ is a negative exponential RV (recall that $h_{1, n}$ is a complex Gaussian RV). Therefore, we obtain the following expression for the conditional outage probability (when we fix the set of RVs $\left\{\chi_{m}, h_{x, m}, \delta_{x, m}\right\}$ ):

$$
\begin{aligned}
& \operatorname{Pr}\left(\operatorname{SINR}_{n}<\zeta_{n} \mid\left\{\chi_{m}, h_{x, m}, \delta_{x, m}\right\}_{x, m}\right) \\
& =1-\exp \left\{-\zeta_{n}\left[\frac{1}{G / n} \sum_{m=1}^{M} \sum_{\substack{x=1, m \neq n \\
x=2, m=n}}^{x_{m}} \chi_{m} s_{m}\left(\operatorname{Re}\left\{h_{x, m}\right\}\right)^{2}\right.\right. \\
& \left.\left.\quad \times\left[\rho^{2}\left(\delta_{x, m}\right)+\hat{\rho}^{2}\left(\delta_{x, m}\right)\right]+\frac{n s_{1}}{2\left(E_{1} / N_{0}\right)}\right]\right\} .
\end{aligned}
$$

When we remove the condition on $\left\{\chi_{m}, h_{x, m}, \delta_{x, m}\right\}_{x, m}$, we obtain

$$
\begin{aligned}
\operatorname{Pr}\left(\operatorname{SINR}_{n}<\zeta_{n}\right) & =e^{-\frac{n s_{1} / s_{n}}{E_{1} / N_{0}} \zeta_{n}} \prod_{m=1}^{M} \prod_{\substack{x=1, m \neq n \\
x=2, m=n}}^{x_{m}} E_{\chi_{m}, h_{x, m}, \delta_{x, m}} \\
\times & {\left[e^{\left.-\zeta_{n}\left(\frac{2 \chi m}{G / n} \frac{s_{m}}{s_{n}}\left(\operatorname{Re}\left\{h_{x, m}\right\}\right)^{2}\left[\rho^{2}\left(\delta_{x, m}\right)+\hat{\rho}^{2}\left(\delta_{x, m}\right)\right]\right)\right] .}\right.}
\end{aligned}
$$

Because, for any $y, E_{\chi_{m}, Y}\left[E\left[e^{-\chi_{m} y} \mid Y=y\right]\right]=1-q_{m}+$ $q_{m} E_{Y}\left[e^{-y}\right]$, the outage probability in (11) is reduced to

$$
\begin{array}{r}
\operatorname{Pr}\left(\operatorname{SINR}_{n}<\zeta_{n}\right)=1-e^{-\frac{n s_{1} / s_{n}}{E_{1} / N_{0}} \zeta_{n}} V^{x_{n}-1}\left(n \zeta_{n}\right) \\
\times \prod_{m=1, m \neq n}^{M} V^{x_{m}}\left(\frac{n s_{m}}{s_{n}} \zeta_{n}\right)
\end{array}
$$

where

$$
\begin{aligned}
V(z)=1-q_{m} & +q_{m} E_{h_{x, m}, \delta_{x, m}} \\
& \times\left[e^{-\frac{2 z}{G}\left(\operatorname{Re}\left\{h_{x, m}\right\}\right)^{2}\left[\rho^{2}\left(\delta_{x, m}\right)+\hat{\rho}^{2}\left(\delta_{x, m}\right)\right]}\right]
\end{aligned}
$$

which depends on the shape of the chip waveform. In the special case of unit rectangular waveform, where $\rho\left(\delta_{x, m}\right)=1-\delta_{x, m}$ and $\hat{\rho}\left(\delta_{x, m}\right)=\delta_{x, m}$, (7) reduces to $\operatorname{Var}\left(\mathfrak{W}_{x, m} \mid\left\{\delta_{x, m}\right\}_{x, m}\right)=$ $1 /(G / n)\left[1+2 \delta_{x, m}\left(\delta_{x, m}-1\right)\right]$ [20]. This allows us to obtain a closed-form expression of $V(z)$ given by

$$
V(z)=1-q_{m}+q_{m}\left[\frac{1}{2} \sqrt{\frac{G}{z}} \ln \left(\frac{\sqrt{G+2 z}+\sqrt{z}}{\sqrt{G+2 z}-\sqrt{z}}\right)\right]
$$

which follows the fact that $\left(\operatorname{Re}\left\{h_{x, m}\right\}\right)^{2}$ is a chi-square RV.

\section{B. QPSK-Modulated System}

A QPSK-modulated signal of class- $m$ can be represented by [21], [22]

$$
\begin{aligned}
v_{x, m}(t) & =h_{x, m} \sqrt{2 s_{m}} \\
& \times \sum_{j=-\infty}^{\infty}\left[b_{j, x, m}^{(I)}(t) a_{x, m}^{(I)}(t)-i b_{j, x, m}^{(Q)}(t) a_{x, m}^{(Q)}(t)\right]
\end{aligned}
$$

where $I$ and $Q$ represent the in-phase and quadrature phase components, respectively. Assume that $a_{x, m}^{(I)}(t)$ and $a_{x, m}^{(Q)}(t)$ are independent signature sequences. Likewise, $b_{j, x, m}^{(I)}(t)$ and $b_{j, x, m}^{(Q)}(t)$ are independent data bit streams, which can be expressed as in (1).

Analogous to the BPSK, the conditional variance of the $j$ th bit class- $n$ decision variable for the in-phase component is obtained, in the case of the rectangular chip waveform, by

$$
\begin{aligned}
& \operatorname{Var}_{Z_{j, 1, n}}\left[Z_{j, 1, n} \mid\left\{\chi_{m}, h_{x, m}, \delta_{x, m}\right\}_{x, m}\right] \\
& =\frac{1}{G / n} \sum_{m=1}^{M} \sum_{\substack{x=1, m \neq n \\
x=2, m=n}}^{x_{m}} \chi_{m} s_{m}\left|h_{x, m}\right|^{2} \\
& \quad \times\left[1+2 \delta_{x, m}\left(\delta_{x, m}-1\right)\right]+\frac{n s_{1}}{2\left(E_{1} / N_{0}\right)} .
\end{aligned}
$$

Notice here that $\left|h_{x, m}\right|^{2}, \forall x$, and $m$ become i.i.d exponential RVs (a consequence of the fact that the channel gains of the $I$ and $Q$ components are complex Gaussian RVs).

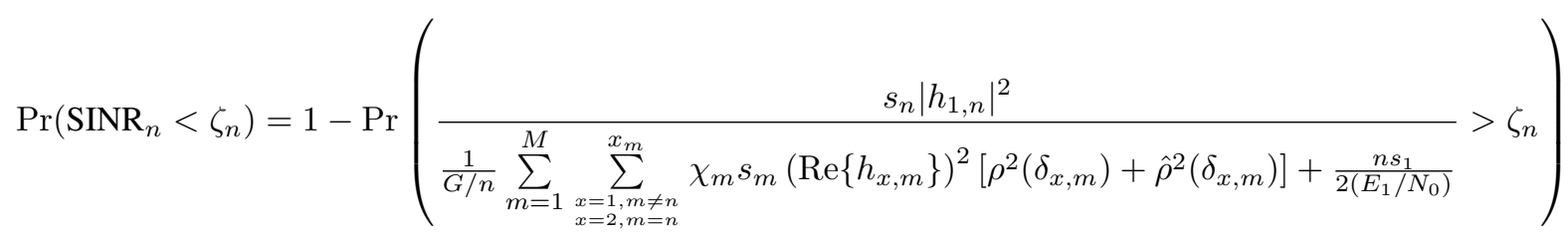




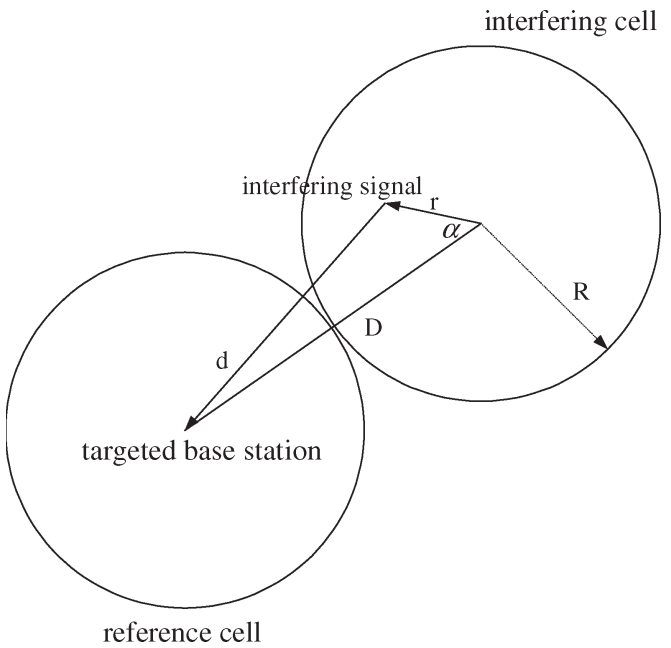

Fig. 1. Uplink propagation model.

When $G \gg 1$, the decision variables converge into a set of independent Gaussian RVs (conditioned on $h_{x, m}, \delta_{x, m}$ ). Accordingly, a class- $n$ SINR is given by (17), shown at the bottom of the page. Similar to (12), the outage probability in this case is

$$
\begin{aligned}
& \operatorname{Pr}\left(\operatorname{SINR}_{n}<\zeta_{n}\right)=1-e^{-\frac{n s_{1} / s_{n}}{E_{1} / N_{0}} \zeta_{n}} V_{Q}^{x_{n}-1}\left(n \zeta_{n}\right) \\
& \times \prod_{m=1, m \neq n}^{M} V_{Q}^{x_{m}}\left(\frac{n s_{m}}{s_{n}} \zeta_{n}\right)
\end{aligned}
$$

where

$V_{Q}(z)=1-q_{m}+q_{m} E_{h_{x, m}, \delta_{x, m}}\left[e^{-\frac{2 z}{G}\left|h_{x, m}\right|^{2}\left[1+2 \delta_{x, m}\left(\delta_{x, m}-1\right)\right]}\right]$.

It can be shown that $V_{Q}(z)$ can be presented in closed form by

$$
V_{Q}(z)=1-q_{m}+q_{m}\left[\frac{G}{\sqrt{z(G+z)}} \tan ^{-1} \sqrt{\frac{z}{G+z}}\right] .
$$

\section{Multicell System Model}

By assuming a circular-shaped cellular system (see Fig. 1), the SINR of a class- $n$ signal is

$$
\operatorname{SINR}_{n}=\frac{1}{2} \frac{s_{n}\left|h_{1, n}\right|^{2}}{\operatorname{Var}_{Z_{j, 1, n}}\left[Z_{j, 1, n}\right]}
$$

Analogous to the single-cell analysis, $\operatorname{Var}_{Z_{j, 1, n}}\left[Z_{j, 1, n} \mid\right.$ $\left.\left\{\chi_{m}, h_{x, m, k}, \delta_{x, m, k}\right\}_{x, m, k}\right]$ is given by

$$
\begin{aligned}
\operatorname{Var}_{Z_{j, 1, n}}\left[Z_{j, 1, n} \mid\left\{\chi_{m}, h_{x, m, k}, \delta_{x, m, k}\right\}_{x, m, k}\right] \\
=\frac{1}{G / n} \sum_{m=1}^{M} \sum_{\substack{x=1, m \neq n \\
x=2, m=n}}^{x_{m}} \chi_{m} s_{m}\left(\operatorname{Re}\left\{h_{x, m}\right\}\right)^{2} \\
\quad \times\left[1+2 \delta_{x, m}\left(\delta_{x, m}-1\right)\right] \\
\quad+\frac{1}{G / n} \sum_{k}^{K} \sum_{m=1}^{M} \sum_{x=1}^{x_{m}} \chi_{m} \mathcal{S}_{x, m, k}\left(\operatorname{Re}\left\{h_{x, m, k}\right\}\right)^{2} \\
\quad \times\left[1+2 \delta_{x, m, k}\left(\delta_{x, m, k}-1\right)\right]+\frac{n s_{1}}{2\left(E_{1} / N_{0}\right)}
\end{aligned}
$$

where $K$ is the number of adjacent cells, $\mathcal{S}_{x, m, k}$ is the received power from the $x$ th class- $m$ interference in the $k$ th cell, and

$$
\mathcal{S}_{x, m, k}=\mathcal{P}_{x, m, k}\left[r^{2}+D^{2}-2 r D \cos \alpha\right]^{\frac{-\beta}{2}} .
$$

Here, $\mathcal{P}_{x, m, k}$ is the transmitted power, $\beta$ is the path loss exponent [30], $D$ is the distance between the centers of two adjacent cells, and $(r, \alpha)$ are the polar coordinates of an arbitrary cochannel interferer (Fig. 1). When users are uniformly distributed over their home cells, $r$ is an RV that has the following distribution [32]:

$$
f(r)= \begin{cases}2 r / R^{2}, & 0<r<R \\ 0, & \text { otherwise }\end{cases}
$$

With the assumption of perfect (slow) power control, all class- $m$ signals are received at their home base stations with the same average (over fading) power $s_{m}, m=1, \ldots, M$. Therefore, the transmitted power from an arbitrary mobile user at distance $r$ from its home cell is $\mathcal{P}_{x, m, k}=s_{m} r^{\beta}$, and

$$
\mathcal{S}_{x, m, k}=s_{m} d^{-\beta}(r, \alpha)
$$

where $d(r, \alpha)=\sqrt{1+(D / r)^{2}-2(D / r) \cos \alpha}$.

To simplify our presentation, assume that all $K$ interfering cells have the same number of users. Consequently, the outage probability is given by

$$
\begin{aligned}
\operatorname{Pr} & \left.\operatorname{SINR}_{n}<\zeta_{n}\right) \\
= & 1-e^{-\frac{n s_{1} / s_{n}}{E_{1} / N_{0}} \zeta_{n}} V^{x_{n}-1}\left(n \zeta_{n}\right) \\
\times & {\left[\prod_{m=1, m \neq n}^{M} V^{x_{m}}\left(\frac{n s_{m}}{s_{n}} \zeta_{n}\right)\right] } \\
& \cdot\left[\prod_{m=1}^{M} U^{x_{m}}\left(\frac{n s_{m}}{s_{n}} \zeta_{n}\right)\right]^{K}, n=1,2, \ldots, M
\end{aligned}
$$

$$
\operatorname{SINR}_{n}=\frac{s_{n}\left|h_{1, n}\right|^{2}}{\frac{2}{G / n} \sum_{m=1}^{M} \sum_{\substack{x=1, m \neq n \\ x=2, m=n}}^{x_{m}} \chi_{m} s_{m}\left|h_{x, m}\right|^{2}\left[1+2 \delta_{x, m}\left(\delta_{x, m}-1\right)\right]+\frac{s_{1} n}{E_{1} / N_{0}}}
$$


where

$$
U(z)=1-q_{m}+q_{m}\left\{\frac{1}{\pi R^{2}} \int_{0}^{2 \pi} \int_{0}^{R} \mathcal{V}\left[z d^{-\beta}(r, \alpha)\right] r d r d \alpha\right\} .
$$

Note that $\mathcal{V}(z)$ is readily given in (14) and (20) for BPSK and QPSK, respectively.

\section{ThroughPUT MAXIMIZATION AND MAC-PHY OPTIMIZATION}

In this section, we present a throughput maximization formula whose optimum solution can be accomplished by a linear programming approach. The proposed formula is used to select the optimum number of transmissions that maximizes the overall average throughput and satisfies the PHY layer constraints. It is also found that we can include the MAC layer constraints, concerning delay, in the formula. This facilitates the construction of the MAC-PHY optimization, which is shown in Section III-B.

\section{A. Throughput Maximization}

Let $x_{1}, x_{2}, \ldots, x_{M} \in \mathcal{I}_{+}$be positive integers that represent the selected transmissions from class- 1 , class $-2, \ldots$, class- $M$, respectively. Then, the maximization problem is formulated by the following integer programming:

$$
\max _{x_{1}, x_{2}, \ldots, x_{M} \in \mathcal{I}_{+}} \sum_{m=1}^{M} \varpi_{m} q_{m} x_{m}
$$

subject to

$$
\operatorname{Pr}\left(\operatorname{SINR}_{m}<\zeta_{m}\right) \leq \varepsilon_{m}, \quad m=1,2, \ldots, M
$$

where $q_{m}$ is the class- $m$ activity probability. $\varepsilon_{m}$ is the maximum class- $m$ outage probability, which is given in (12) and (18) for BPSK and QPSK, respectively. $\varpi_{m}$ is an arbitrary positive number that represents the weight of a class- $m$ transmission.

Here, we suggest to simplify (28) by letting $x_{1}, \ldots, x_{M} \in$ $\mathcal{R}_{+}$be the positive real numbers instead of integers. Without any significant error, (28) can be approximated by a linear programming problem.

As far as the constraint in the optimization formula in (28) is concerned, notice from (12) or (18) that we can transform the nonlinear constraint, in Rayleigh fading, into an equivalent set of linear inequalities by taking the natural logarithm of both sides. Then

$\sum_{m=1}^{M} x_{m} \ln \left[V\left(\frac{n s_{m}}{s_{n}} \zeta_{n}\right)\right] \geq \ln \left[\left(1-\varepsilon_{m}\right) V\left(n \zeta_{n}\right)\right]+\frac{n s_{1} / s_{n}}{E_{1} / N_{0}} \zeta_{n}$

which can be arranged in the matrix form

$$
\mathrm{Ax} \leq \varsigma
$$

where $\mathbf{x}=\left[x_{1}, x_{2}, \ldots, x_{M}\right]^{T}, \mathbf{A}$ is the $M \times M$ coefficient matrix with elements given by

$$
v_{m n}=-\ln \left[V\left(\frac{m s_{n}}{s_{m}} \zeta_{m}\right)\right], \quad n, m=1,2, \ldots, M
$$

and $\varsigma=\left[\varsigma_{1}, \varsigma_{2}, \ldots, \varsigma_{M}\right]^{T}$ with each element $\varsigma_{m}$ expressed as

$$
\begin{aligned}
\varsigma_{m}=-\ln \left[\left(1-\varepsilon_{m}\right)\right. & \left.V\left(m \zeta_{m}\right)\right] \\
& -\frac{m s_{1} / s_{m}}{E_{1} / N_{0}} \zeta_{m}, \quad m=1,2, \ldots, M
\end{aligned}
$$

where $V(z)$ is readily given in (14) and (20) for BPSK and QPSK, respectively.

In the case of a multicell scenario, (26) can be transformed into a set of linear inequalities and rearranged in the matrix form $\mathbf{A x} \leq \boldsymbol{\varsigma}$, where the elements $v_{m n}$ in matrix $\mathbf{A}$ are given by

$$
\begin{array}{r}
v_{m n}=-\left\{\ln \left[V\left(\frac{m s_{n}}{s_{m}} \zeta_{m}\right)\right]+6 \ln \left[U\left(\frac{m s_{n}}{s_{m}} \zeta_{m}\right)\right]\right\} \\
n, m=1,2, \ldots, M
\end{array}
$$

and the elements $\varsigma_{m}$ still have the same expression as in (32).

Accordingly, the new linear programming for the throughput maximization is given by

$$
\max _{x_{1}, x_{2}, \ldots, x_{M} \in \mathcal{R}_{+}} \sum_{m=1}^{M} \varpi_{m} q_{m} x_{m}
$$

subject to

$$
\begin{aligned}
& \mathbf{A x} \leq \boldsymbol{\varsigma} \\
& x_{m} \geq 0, \quad m=1,2, \ldots, M .
\end{aligned}
$$

It is worth saying that the last line of the constraints can be used as the upper and lower limits of the individual class- $m$ transmissions, i.e.,

$$
\sigma_{m} x_{m, \max } \leq x_{m} \leq x_{m, \max }, \quad m=1,2, \ldots, M
$$

where $x_{m, \max }$ is an arbitrary upper bound of the transmissions. $\sigma_{m} \in(0,1]$ represents the transmission limit factor that can be used to provide some degrees of fairness transmission. As a matter of fact, based on the results of our previous work [9], the upper and lower bound in (35) are very essential for multirate communication because the scheduler prefers to allow the high-rate, rather than the low-rate, users to transmit their information. This is due to the fact that the high-rate transmissions offer more reward to the cost function, as well as to the system throughput.

In Section III-B, we will show that the proposed throughput maximization can be related to the MAC-PHY optimization by adding some MAC constraints.

\section{B. MAC-PHY Optimization}

Let us consider a MAC layer model of a VSG-CDMA uplink (Fig. 2). There are a total of $M$ classes (rates) of transmissions originating from the users within the cell. At the base station, all 


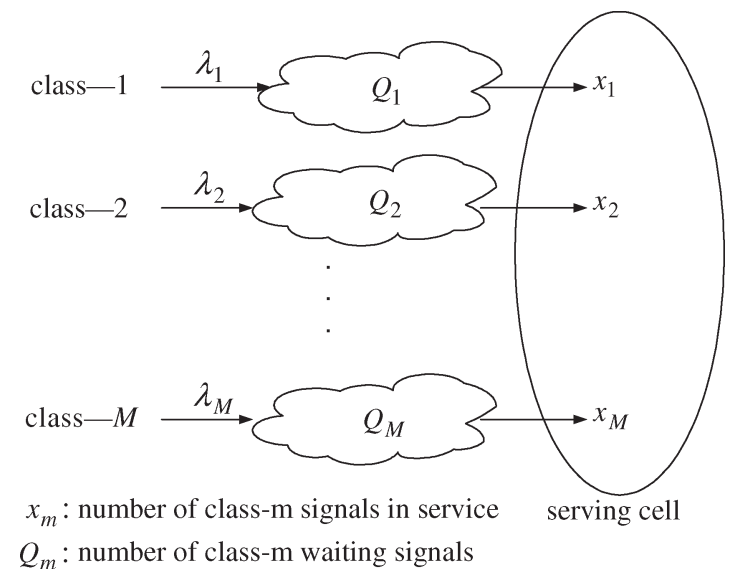

Fig. 2. MAC layer model.

requests for the next uplink are accommodated into $M$ groups according to the different transmission rates. The requests in each group are served on the order of first-come, first-served discipline, and the base station is responsible for selecting the appropriate number of transmissions from individual groups for the next uplink. In the case where the base station cannot serve all the requests, the remainder has to wait for the following deliveries. This results in a waiting time delay, which is the significant issue of MAC design. In this paper, we handle this problem by applying Little's law [25] as follows. Let us consider the number of pending transmissions of class- $m$ as

$$
Q_{m}=\lambda_{m} W_{m}
$$

where $\lambda_{m}$ is the class- $m$ arrival rate, and $W_{m}$ is the average waiting time per transmission.

Define $W_{m, \max }$ as the maximum tolerable delay before the corresponding request of transmission is out of date (and thus discarded). Let $X_{m} \equiv \lambda_{m} W_{m, \max }$ be the maximum queue length [29]. We assume that no requests can stay in the queue longer than $W_{m, \max }$. Consequently, the number of pending requests $Q_{m}$ is bounded by $X_{m}$ given by

$$
Q_{m} \leq X_{m}
$$

As a result, we can formulate the MAC-PHY optimization problem as

$$
\max _{x_{1}, x_{2}, \ldots, x_{M} \in \mathcal{R}_{+}} \sum_{m=1}^{M} \varpi_{m} q_{m} x_{m}
$$

subject to

$$
\begin{aligned}
\mathbf{A} \mathbf{x} & \leq \boldsymbol{\varsigma} \\
\mathbf{X}_{\min } & \leq \mathbf{x} \leq \mathbf{X}_{\max }
\end{aligned}
$$

where $\mathbf{X}_{\max }=\left[X_{1}, X_{2}, \ldots, X_{M}\right]^{T}$, and $\mathbf{X}_{\min }=\left[\sigma_{1} X_{1}\right.$, $\left.\sigma_{2} X_{2}, \ldots, \sigma_{M} X_{M}\right]^{T}$.

Assume that there is high uplink traffic demand. Therefore, only $x_{m}$ transmissions are served per round, whereas

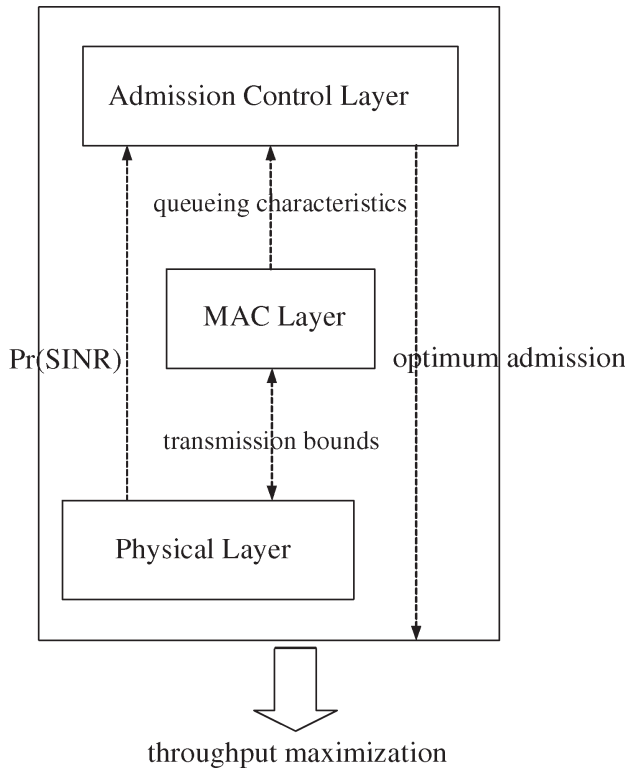

Fig. 3. Cross-layer interaction.

$X_{m}-x_{m}$ transmissions are left waiting. To minimize the number of pending transmissions, we can alternatively formulate the cost function by

$$
X_{m}-x_{m}, \quad m=1,2, \ldots, M
$$

which forms a linear minimization problem that is equivalent to (38) and is expressed as

$$
\min _{x_{1}, x_{2}, \ldots, x_{M} \in \mathcal{R}_{+}} \sum_{m=1}^{M} \varpi_{m} q_{m}\left(X_{m}-x_{m}\right)
$$

subject to

$$
\begin{aligned}
\mathbf{A x} & \leq \boldsymbol{\varsigma} \\
\mathbf{X}_{\min } & \leq \mathbf{x} \leq \mathbf{X}_{\text {max }} .
\end{aligned}
$$

\section{Optimal AdmisSion CONTROL}

In this section, we present an optimal admission policy for the cross-layer cooperation in Fig. 3. First, a new admissible state space is determined and used as a boundary to control the number of admitted users. Then, the admission process and the resource sharing strategy are described. Finally, the optimal policy is formulated by a linear programming approach.

\section{A. Admissible Region}

Let us consider the capacity of a single-cell BPSK-modulated CDMA system as a pool of $C$ resource units. Let $\beta_{m}, m=$ $1, \ldots, M$ be the required capacity per class- $m$ transmission. Assume that the new arrivals are admitted in the system as long as the sum of the used capacity does not exceed the total 
capacity. This admission policy is called complete sharing, whose admission region is given by [26]

$$
\Phi=\left\{\mathbf{x}=\left(x_{1}, x_{2}, \ldots, x_{M}\right) \in \mathcal{I}_{+}^{M}: \sum_{m=1}^{M} \beta_{m} x_{m} \leq C\right\}
$$

where $\mathcal{I}_{+}^{M}$ represents non-negative $M$-dimensional integers, and $x_{m}$ is the number of class- $m$ signals.

Let $x_{m, \max }=\left\lfloor C / \beta_{m}\right\rfloor$ [26] be the maximum number of class- $m$ transmissions that can be accommodated when the whole system capacity is used only by class- $m$ transmissions. Then, (41) can be converted to

$$
\Phi=\left\{\left(x_{1}, \ldots, x_{M}\right) \in \mathcal{I}_{+}^{M}: \sum_{m=1}^{M} \frac{x_{m}}{x_{m, \max }} \leq 1\right\}
$$

As far as $\left\{x_{m, \max }\right\}, m=1, \ldots, M$ are concerned, we can express $x_{m, \max }$ by (12) as follows. Letting all $x_{n}=0$ for $n \neq$ $m$, we then have

$$
\begin{aligned}
\operatorname{Pr}\left(\operatorname{SINR}_{m}<\zeta_{m}\right) & =1-e^{-\frac{m s_{1} / s_{m}}{E_{1} / N_{0}} \zeta_{m}} V^{x_{m, \max }-1}\left(m \zeta_{m}\right) \\
& \leq \varepsilon_{m}, \quad m=1,2, \ldots, M
\end{aligned}
$$

By taking the natural logarithm of both sides of (43), $x_{m, \max }$ is given by

$$
x_{m, \max }=\frac{\left[\ln \left(1-\varepsilon_{m}\right)+\frac{m s_{1} / s_{m}}{E_{1} / N_{0}} \zeta_{m}\right]}{\ln \left[V\left(m \zeta_{m}\right)\right]}+1 .
$$

As a result, the admission region is transformed into

$$
\begin{aligned}
\Phi=\{( & \left(x_{1}, \ldots, x_{M}\right) \in \mathcal{I}_{+}^{M}: \\
& \left.\sum_{m=1}^{M} \frac{x_{m} \ln \left[V\left(m \zeta_{m}\right)\right]}{\ln \left[\left(1-\varepsilon_{m}\right) V\left(m \zeta_{m}\right)\right]+\frac{m s_{1} / s_{m}}{E_{1} / N_{0}} \zeta_{m}} \leq 1\right\}
\end{aligned}
$$

where $V(z)$ is given in (14) and (20) for BPSK and QPSK, respectively.

Analogous to the single-cell analysis, the admission region of a multicell CDMA uplink is determined by applying (26) to the procedure shown in (41)-(45). As a result, the outage probability for a multicell system is

$$
\begin{aligned}
& \Phi_{\text {multicell }}=\left\{\left(x_{1}, \ldots, x_{M}\right) \in \mathcal{I}_{+}^{M}:\right. \\
&\left.\sum_{m=1}^{M} x_{m}\left(\frac{\ln \left[V\left(m \zeta_{m}\right)\right]+6 \ln \left[U\left(m \zeta_{m}\right)\right]}{\ln \left[\left(1-\varepsilon_{m}\right) V\left(m \zeta_{m}\right)\right]+\frac{m s_{1} / s_{m}}{E_{1} / N_{0}} \zeta_{m}}\right) \leq 1\right\}
\end{aligned}
$$

where $U(z)$ is previously defined in (27), and $x_{m}$ represents the number of class- $m$ signals in each cell.

\section{B. System States, Decision Epochs, and State Dynamics}

The admission control problem is characterized as an SMDP process that can be explained as follows. At the base station (Fig. 2), a new class- $m$ arrival is accommodated into the corresponding virtual queue. The queueing system is modeled as $\mathrm{M} / \mathrm{M} / x_{m} / X_{m}$ [25], where $x_{m}$ represents the number of signals in service, which is determined by the admission region in (45). $X_{m} \geq x_{m}+Q_{m}$ is the limit of admitted signals that is equivalent to (37), where $Q_{m}$ indicates the number of signals waiting for service.

Given a dynamic system, which is monitored at time instances (epochs), at each epoch, the system is classified into one of the possible states, and subsequently, a decision is made. Let $\overline{\mathbf{x}}_{m}=\left[x_{m}, Q_{m}\right]$ be a 2-D vector. Then, the state space of the system is

$$
\begin{aligned}
\Psi_{\overline{\mathbf{x}}}=\left\{\overline{\mathbf{x}}: \overline{\mathbf{x}}=\left(\overline{\mathbf{x}}_{1}, \ldots, \overline{\mathbf{x}}_{M}\right): x_{m} \in \Phi\right. & \\
& \left.x_{m}+Q_{m} \leq X_{m}, \quad m=1,2, \ldots, M\right\}
\end{aligned}
$$

where $\Phi$ is the admission region given in (45).

At the decision epoch $t$, when a new signal arrives at the system with the existing signals in state $\overline{\mathbf{x}}(t)$, there are two actions to be chosen for the new arrival, i.e., accept or reject. Because our admission model has included the queueing issue, the action space can be differently defined from the literature (e.g., [29] and [33]) as

$$
\mathbf{A}=\left\{\mathbf{a}: \mathbf{a}=\left(a_{1}, \ldots, a_{M}\right): a_{m} \in\{0,1\}, m \in 1,2, \ldots, M\right\}
$$

where

$$
a_{m}= \begin{cases}1, & \text { if } x_{m} \in \Phi \\ 0, & \text { otherwise. }\end{cases}
$$

Note that $\mathbf{a} \neq(0,0, \ldots, 0)$ when $\overline{\mathbf{x}}=(0,0, \ldots, 0)$; otherwise, new connections are never admitted into the network, and the system cannot evolve.

When the capacity is fully exploited by the existing signals, a new class- $m$ arrival could be admitted into the system as long as the number of served and waiting signals is less than the admission limit, i.e., $x_{m}+Q_{m} \leq X_{m}$. Otherwise, the new incoming signal is blocked (if $a_{m}=0$ and $x_{m}+Q_{m}>X_{m}$ ).

It is common to model the dynamics of a multirate CDMA system by an SMDP embedded at the expected sojourn time, which is defined as the shortest time interval between two consecutive events (e.g., [27]), given by

$$
\begin{aligned}
\tau_{\overline{\mathbf{x}} \mathbf{a}}=\left[\sum _ { m = 1 } ^ { M } \left\{x_{m} \mu_{m}+Q_{m} a_{m} \mu_{m}+\lambda_{m} a_{m}\right.\right. \\
\left.\left.\quad+\lambda_{m}\left(1-a_{m}\right) \mathrm{u}\left[X_{m}-\left(x_{m}+Q_{m}\right)\right]\right\}\right]^{-1} .
\end{aligned}
$$

with $\lambda_{m}$ and $\mu_{m}$ being the average class- $m$ arrival and departure rate, respectively. Notice that a new signal is blocked when $\left(1-a_{m}\right) \mathrm{u}\left[X_{m}-\left(x_{m}+Q_{m}\right)\right]=0$, where $\mathrm{u}[\cdot]$ is the unit step function. 
The state transition probabilities are defined as

$$
\begin{aligned}
& P(\overline{\mathbf{y}} \mid \overline{\mathbf{x}}, \mathbf{a}) \\
& = \begin{cases}\lambda_{m} a_{m} \tau_{\overline{\mathbf{x}} \mathbf{a}}, & \text { if } \overline{\mathbf{y}}_{m}=\overline{\mathbf{x}}_{m}+e_{m} \\
\lambda_{m}\left(1-a_{m}\right) \mathrm{u} & \\
\times\left[X_{m}-\left(x_{m}+Q_{m}\right)\right] \tau_{\overline{\mathbf{x}} \mathbf{a}}, & \text { if } \overline{\mathbf{y}}_{m}=\overline{\mathbf{x}}_{m}+e_{m}^{(Q)} \\
x_{m} \mu_{m} \tau_{\overline{\mathbf{x}} \mathbf{a}}, & \text { if } \overline{\mathbf{y}}_{m}=\overline{\mathbf{x}}_{m}-e_{m} \\
Q_{m} a_{m} \mu_{m} \tau_{\overline{\mathbf{x}} \mathbf{a}}, & \text { if } \overline{\mathbf{y}}_{m}=\overline{\mathbf{x}}_{m}-e_{m}^{(Q)} \\
0, & \text { otherwise }\end{cases}
\end{aligned}
$$

where $m=1, \ldots, M . e_{m}=[1,0]$ and $e_{m}^{(Q)}=[0,1]$ are 2-D vectors that represent the transition of the current state $\overline{\mathbf{x}}_{m}$ to the next state $\overline{\mathbf{y}}_{m}$. For example, given $\overline{\mathbf{x}}_{m}=\left[x_{m}, Q_{m}\right]$, if there is a class- $m$ user arriving at queue $m$, then $\overline{\mathbf{y}}_{m}=\left[x_{m}, Q_{m}+\right.$ 1] $=\overline{\mathbf{x}}_{m}+e_{m}^{(Q)}$.

It is to be noticed that, in contrast to [27], in (50), for the sojourn time, and in (51), for the transition probability, we have included the terms $Q_{m} a_{m} \mu_{m}$ and $\lambda_{m}\left(1-a_{m}\right) \mathrm{u}\left[X_{m}-\left(x_{m}+\right.\right.$ $\left.\left.Q_{m}\right)\right]$ in order to account for the departure and arrival on queue $m, m=1, \ldots, M$.

\section{Admission Control Optimization}

The cross-layer optimization is obtained by solving the following linear programming:

$$
\max \sum_{\overline{\mathbf{x}} \in \Psi_{\overline{\mathbf{x}}}} \sum_{\mathbf{a} \in \mathbf{A}_{\overline{\mathbf{x}}}} \sum_{m \in M} \varpi_{m} q_{m} x_{m} \tau_{\overline{\mathbf{x}} \mathbf{a}} u_{\overline{\mathbf{x}} \mathbf{a}}
$$

subject to

$$
\begin{aligned}
& \sum_{\mathbf{a} \in \mathbf{A}_{\overline{\mathbf{y}}}} u_{\overline{\mathbf{y}} \mathbf{a}}-\sum_{\overline{\mathbf{x}} \in \Psi_{\overline{\mathbf{x}}}} \sum_{\mathbf{a} \in \mathbf{A}_{\overline{\mathbf{x}}}} P(\overline{\mathbf{y}} \mid \overline{\mathbf{x}}, \mathbf{a}) u_{\overline{\mathbf{x}} \mathbf{a}}=0 \\
& \sum_{\overline{\mathbf{x}} \in \Psi_{\overline{\mathbf{x}}}} \sum_{\mathbf{a} \in \mathbf{A}_{\overline{\mathbf{x}}}} \tau_{\overline{\mathbf{x}} \mathbf{a}} u_{\overline{\mathbf{x}} \mathbf{a}}=1 \\
& \sum_{\overline{\mathbf{x}} \in \Psi_{\overline{\mathbf{x}}}} \sum_{\mathbf{a} \in \mathbf{A}_{\overline{\mathbf{x}}}}\left(1-a_{m}\right)\left\{1-\mathbf{u}\left[X_{m}-\left(x_{m}+Q_{m}\right)\right]\right\} \\
& \times \tau_{\overline{\mathbf{x}} \mathbf{a}} u_{\overline{\mathbf{x}} \mathbf{a}} \leq \gamma_{m}, \quad m=1,2, \ldots, M \\
& \sigma_{m} X_{m} \leq \sum_{\overline{\mathbf{x}} \in \Psi_{\overline{\mathbf{x}}} \sum_{\mathbf{a} \in \mathbf{A}_{\overline{\mathbf{x}}}} x_{m} \tau_{\overline{\mathbf{x}} \mathbf{a}} u_{\overline{\mathbf{x}} \mathbf{a}} \leq X_{m}, \quad m=1,2, \ldots, M} \\
& u_{\overline{\mathbf{x}} \mathbf{a}} \geq 0 ; \quad \mathbf{\mathbf { x }} \in \Psi_{\overline{\mathbf{x}}}, \quad \mathbf{a} \in \mathbf{A}_{\overline{\mathbf{x}}}
\end{aligned}
$$

where $\varpi_{m}$ is the reward of class- $m$ transmissions, and $\gamma_{m}$ is the maximum threshold of class- $m$ blocking probability. $u_{\overline{\mathbf{x}} \mathbf{a}}$ is the long-run fraction of decision epochs at which the system is in state $\overline{\mathbf{x}}$ and action a is made [33]. The first two equations hold the state equilibrium. The third and fourth constraints stand for blocking probability and transmission limits, respectively.

Note that we can replace $q_{m} x_{m}$ in (52) with

$$
\left(1-a_{m}\right)\left\{1-\mathrm{u}\left[X_{m}-\left(x_{m}+Q_{m}\right)\right]\right\} .
$$

This leads to a minimization problem, which is given by

$$
\begin{aligned}
\min \sum_{\overline{\mathbf{x}} \in \Psi_{\overline{\mathbf{x}}}} \sum_{\mathbf{a} \in \mathbf{A}_{\overline{\mathbf{x}}}} \sum_{m \in M} \varpi_{m}\left(1-a_{m}\right) \\
\quad \times\left\{1-\mathbf{u}\left[X_{m}-\left(x_{m}+Q_{m}\right)\right]\right\} \tau_{\overline{\mathbf{x}} \mathbf{a}} u_{\overline{\mathbf{x}} \mathbf{a}}
\end{aligned}
$$

subject to the constraints in (52).

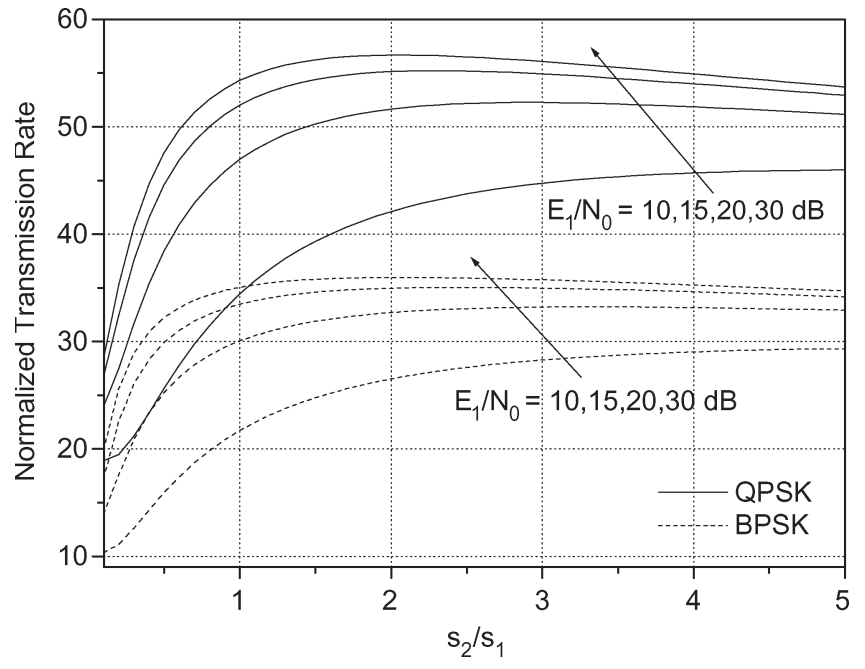

Fig. 4. Effect of channel noise $\left(E_{1} / N_{0}\right)$. Optimum throughput versus $s_{2} / s_{1}$ with targeted SINR $=2 \mathrm{~dB}, G=500, r_{2}=2 r_{1}$, and $\varepsilon_{1}=\varepsilon_{2}=0.01$.

\section{NUMERICAL RESULTS AND DISCUSSIONS}

The numerical presentation consists of two major parts, namely the MAC-PHY optimization and the optimal crosslayer (CAC-MAC-PHY) admission control, as follows.

\section{A. MAC-PHY Optimization}

Here, we use the MAC-PHY optimization formula proposed in (38) to study the impact of transmit power level and MAClayer delay constraints on the optimum throughput. At the end of this section, we will show that (38) can be applied as a useful formula to select an optimal number of data users in VSGintegrated voice and data systems.

1) Optimal Power Selection: Consider a VSG-CDMA dual-class system in the case of QPSK and BPSK. $s_{2} / s_{1}$ is the power ratio, and $r_{2} / r_{1}$ is the rate ratio of class- 2 over class- 1 . Denote the class- 1 rate by the basic rate and the class- 2 by the high rate. The other parameters are $G=500$ and targeted SINR $\zeta=2 \mathrm{~dB}$.

Fig. 4 is concerned with the impact of channel noise (represented by $E_{1} / N_{0}$ ) on the optimal selection of transmitted powers. In the figure, the throughput (normalized transmission rate) is plotted against the power ratio $s_{2} / s_{1}$ for $r_{2}=2 r_{1}$ and for different values of bit energy to Gaussian noise spectral density ratios $E_{1} / N_{0}=10,15,20,30 \mathrm{~dB}$, with $E_{1}=s_{1} / r_{1}$ being the transmit bit energy of class- 1 .

As far as the transmit power allocations among the different classes are concerned, it is worth mentioning that most of the previous research allocates the transmit powers such that all classes would experience identical bit energies, i.e., $E_{1}=$ $s_{1} / r_{1}=s_{2} / r_{2}=s_{3} / r_{3}=\ldots$ (e.g., [10]-[12]).

However, Fig. 4 reveals that this constant energy power allocation seems to be optimal only at high bit energies to background noise (high $E_{1} / N_{0}$ ). Otherwise, the optimal transmit allocations somewhat depend on the change of $E_{1} / N_{0}$, which reflects the amount of background channel noise. Then, the optimal transmit power should be higher at low $E_{1} / N_{0}$. For example, at $E_{1} / N_{0}=15 \mathrm{~dB}$, optimum $s_{2} / s_{1}=3.0$; then, 


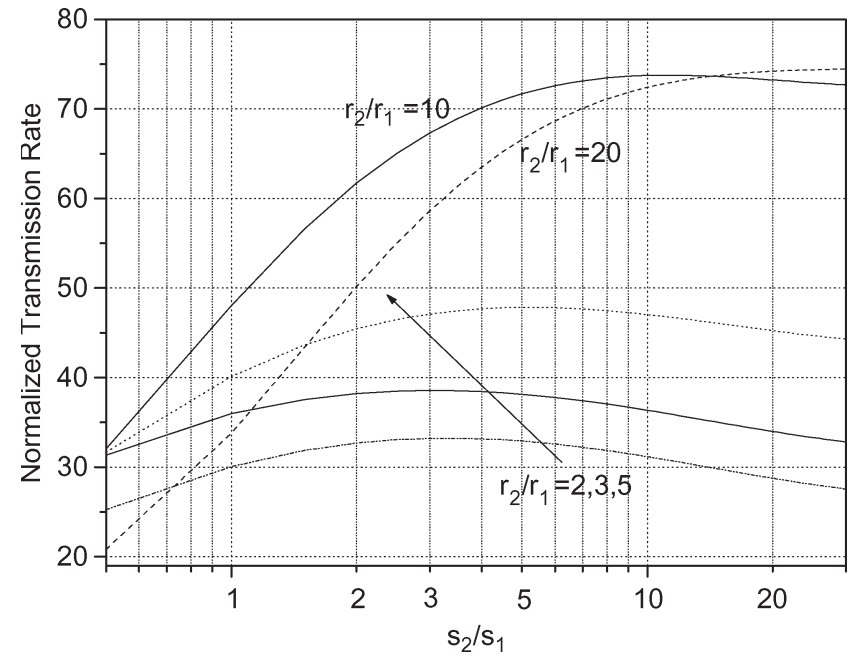

Fig. 5. Effect of rate ratio $\left(r_{2} / r_{1}\right)$. Optimum throughput versus $s_{2} / s_{1}$ in a BPSK-based VSG-CDMA with targeted SINR $=2 \mathrm{~dB}, E_{1} / N_{0}=15 \mathrm{~dB}$, and $\varepsilon_{1}=\varepsilon_{2}=0.01$.

$E_{2, \mathrm{opt}}=1.5 E_{1}$. However, at $E_{1} / N_{0}=10 \mathrm{~dB}$, optimum $s_{2} / s_{1}=4.0$; then, $E_{2, \text { opt }}=2 E_{1}$. Observe that, for $E_{1} / N_{0} \geq$ $20 \mathrm{~dB}$, the optimum ratio tends to be around the rate ratio. In this case, each user would experience the same amount of bit energy, i.e., $E_{2, \text { opt }}=E_{1}=s_{1} / r_{1}=s_{2} / r_{2}$.

In Fig. 5, we plot the throughput against the power ratio $s_{2} / s_{1}$ for several rate ratios $r_{2} / r_{1}=2,3,5,10,20$ at $E_{1} / N_{0}=$ $15 \mathrm{~dB}$. We observe that the constant energy power allocation is optimal at a high rate ratio, e.g., $r_{2} / r_{1} \geq 10$. On the other hand, the power ratio $s_{2} / s_{1}$ is suggested to increase. For instance, at $r_{2} / r_{1}=2$, optimum $s_{2} / s_{1}=3.0$; then, $E_{2, \text { opt }}=1.5 E_{1}$. At $r_{2} / r_{1}=3$, optimum $s_{2} / s_{1}=4.0$, and then, $E_{2, \text { opt }}=1.33 E_{1}$.

2) MAC Layer Constraints: As mentioned in Section III-B, we can relate the last line of the constraints in (38) to the QoS requirements of some higher layers, e.g., MAC layer. Then, we have

$$
\sigma_{m} X_{m} \leq x_{m} \leq X_{m}, \quad m=1,2
$$

where $x_{m}$ is the number of transmitted packets. $X_{m}$, which is the upper bound of transmission, is limited here by the buffer size. $\sigma_{m}$ is defined as a transmission limit factor, which is used to arbitrarily control the minimum transmission of class- $m$.

Let us again consider a BPSK-modulated VSG-CDMA dualclass system with $G=500, E_{1} / N_{0}=15 \mathrm{~dB}$, targeted SINR $\zeta=2 \mathrm{~dB}, \varepsilon_{1}=\varepsilon_{2}=0.01, X_{1}=X_{2}=10$, and $\sigma_{1}, \sigma_{2} \in[0,1]$. To simplify the presentation, assume $s_{2} / s_{1}=r_{2} / r_{1}$.

Fig. 6 is concerned with the issue of fairness transmissions for a multirate CDMA system. Without the lower bound of class- 1 and class- 2 transmissions $\left(\sigma_{1}=\sigma_{2}=0\right)$, the optimal scheduler allocates around $80 \%$ of the total bandwidth (rate) to class-2 traffic (see the solid curves). According to this, the overall throughput would be maximized, but the fairness problem of the low-rate (class-1) transmissions could occur. To reduce such a problem, the constraints in (55) are applied (see the dot curves). With the appropriate values of the lower bound transmissions (by letting $\sigma_{1}=0.5$ and $\sigma_{2}=0.2$ ), the throughput of class- 1 increases to $40 \%$, and thus, the problem of

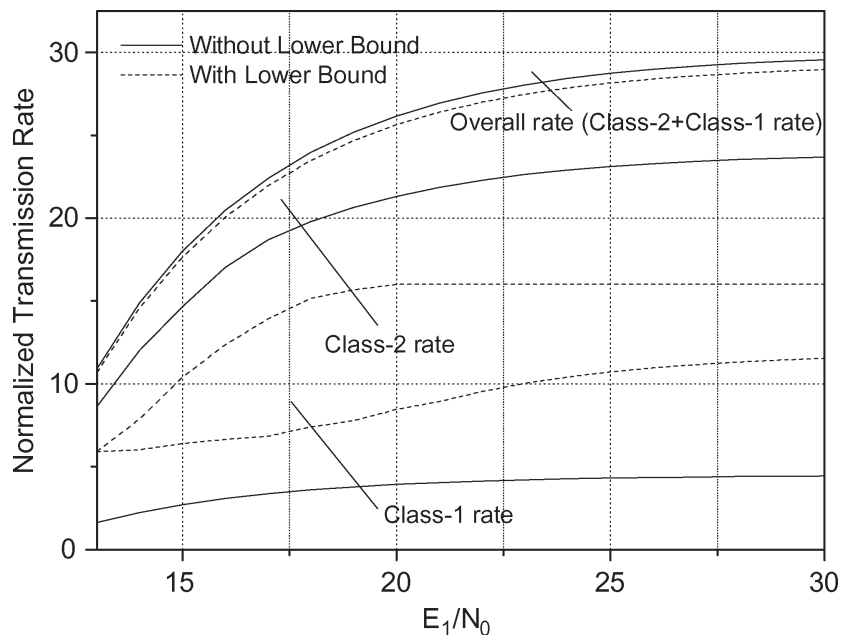

Fig. 6. Minimum transmission constraint. Optimum throughput versus $E_{1} / N_{0}$ in a BPSK-based VSG-CDMA with targeted SINR $=2 \mathrm{~dB}, G=500$, $\varepsilon_{1}=\varepsilon_{2}=0.01, s_{2}=2 s_{1}, \sigma_{1}=0.5, \sigma_{2}=0.2$, and $X_{1}=X_{2}=10$.

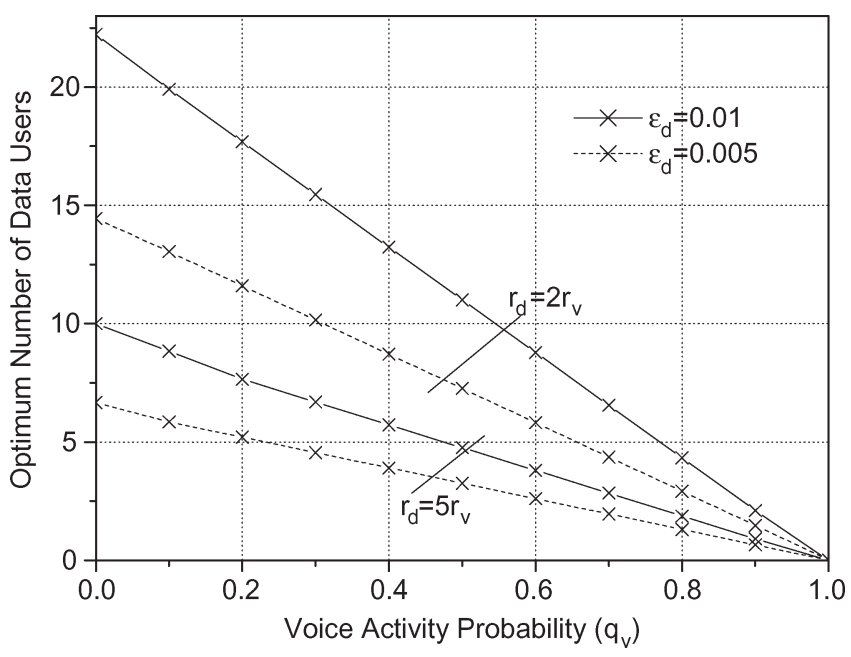

Fig. 7. Optimal selection of number of data users in a BPSK-based VSGCDMA. Targeted SINR $=2 \mathrm{~dB}, G=500, E_{1} / N_{0}=15 \mathrm{~dB}$, and $x_{v}=50$.

fairness is alleviated. However, one should keep in mind that the compensation of low-rate transmissions could lead to a penalty on overall optimum throughput. From Fig. 6, it is found that the overall optimum throughput (total class- 2 + class- 1 rate) reduces when the lower bound constraints are included.

3) Integrated Voice and Data VSG-CDMA: Let us consider an integrated voice and data BPSK-modulated VSG-CDMA system. Because voice is regarded as a real-time traffic, its transmissions always take priority over data in terms of resource allocation.

We fix the number of voice users at 50 and obtain the optimum number of data users by (38). Although the number of voice users is kept constant, their interference is an RV because of the activity probability $q_{v} \in[0,1]$. Let $G=500$, $E_{1} / N_{0}=15 \mathrm{~dB}$, targeted SINR $\zeta=2 \mathrm{~dB}, r_{d}=2 r_{v}$, and $\left(s_{d} / s_{v}\right) /\left(r_{d} / r_{v}\right)=1$, where the subscripts $v$ and $d$ represent voice and data, respectively.

In Fig. 7, we plot the optimum number of data users versus $q_{v}$ for different outage probability constraints and rate ratios. It is obvious that the optimum number of existing data users 


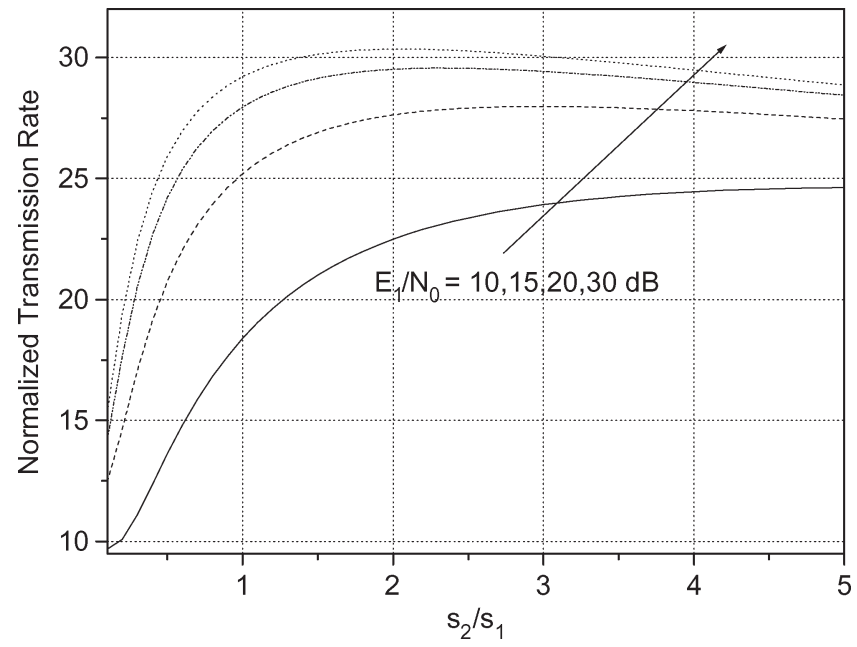

Fig. 8. Multicell (effect of channel noise). Optimum throughput versus $\left(s_{2} / s_{1}\right)$ in a BPSK-based VSG-CDMA with targeted SINR $=2 \mathrm{~dB}, \beta=4$, $G=500, \varepsilon_{1}=\varepsilon_{2}=0.01$, and $r_{2}=2 r_{1}$.

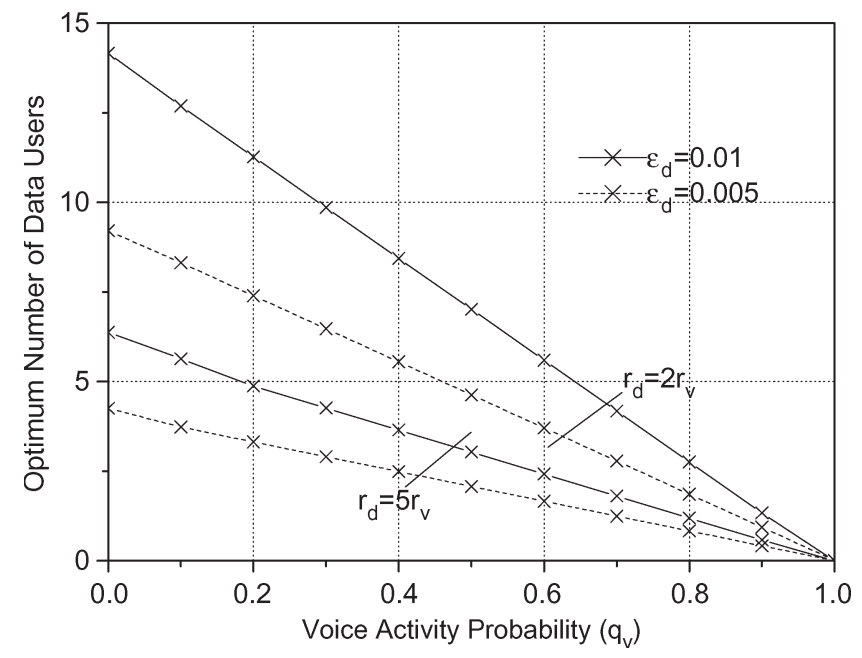

Fig. 9. Multicell. Optimal selection of number of data users in a BPSK-based VSG-CDMA. Targeted SINR $=2 \mathrm{~dB}, G=500, \beta=4, E_{1} / N_{0}=15 \mathrm{~dB}$, and $x_{v}=50$.

linearly goes down against the increase of voice load (which is represented by $q_{v}$ ). These curves can be used to select the optimum number of data users. For example, at voice load, $q_{v}=$ $0.4, x_{v}=50, r_{d}=2 r_{v}$, and the optimum number of data users is 13 , each of which has the maximum allowance of the outage probability $\varepsilon_{d}=0.01$

4) Multicell VSG-CDMA: Consider a BPSK-modulated dual-class VSG-CDMA system, and let $G=500, r_{2}=2 r_{1}$, targeted SINR $\zeta=2 \mathrm{~dB}$, and $\beta=4$.

In Fig. 8, the power ratio $\left(s_{2} / s_{1}\right)$ is plotted with the throughput for a different $E_{1} / N_{0}$ in a multicell environment. Similar to the single-cell case, the constant energy power allocation is optimal only at high $E_{1} / N_{0}$. Otherwise, it is recommended to increase. For instance, at $E_{1} / N_{0}=20 \mathrm{~dB}$, optimum $s_{2} / s_{1}=$ 2.0 , whereas at $E_{1} / N_{0}=10 \mathrm{~dB}$, optimum $s_{2} / s_{1}=4.0$.

Fig. 9 demonstrates the optimal selection on the number of data users. Let the system parameters be the same as Fig. 7 in Section V-A3 (for the single-cell case). Analogously, the optimum number of data users linearly reduces with the increasing

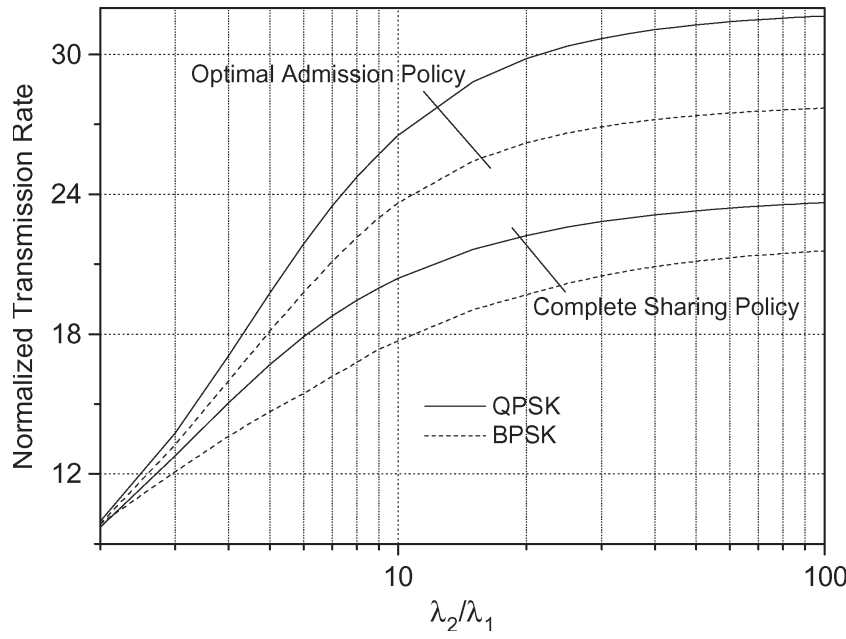

Fig. 10. Optimal admission policy versus complete sharing policy. Throughput plotted with $G=500$, targeted SINR $=2 \mathrm{~dB}, E_{1} / N_{0}=15 \mathrm{~dB}, \varepsilon_{1}=$ $\varepsilon_{2}=0.01, r_{2}=2 r_{1},\left(s_{2} / s_{1}\right) /\left(r_{2} / r_{1}\right)=1, \lambda_{1}=\mu_{1}=\mu_{2}=1$, and $\gamma_{1}=$ $\gamma_{2}=0.1$.

voice activity probability. However, because the intercell interference is included, the optimum number of existing users (for multicell) is less than that of the single-cell case by around $25 \%$.

\section{B. CAC-MAC-PHY Optimization}

1) Optimal Admission Policy: This subsection is concerned with the joint optimal admission policy over three layers-the PHY, MAC, and CAC layers. The optimization formula in (52) is used where the optimum solutions are obtained by the simplex method. To evaluate the system performance of the proposed optimal policy, we compare it with that of the conventional complete sharing policy (see [26] and [33]) in which the total resource is fully shared by all existing users in the system.

The admission region of both policies is given by (45). Here, we measure the performance in terms of overall average throughput and blocking probability. Consider a dual-class single-cell VSG-CDMA system in the case of BPSK and QPSK with the system parameters (categorized by layer)

$$
\begin{aligned}
\text { PHY Layer : } & G=500, \text { targeted } \operatorname{SINR} \zeta=2 \mathrm{~dB} \\
& E_{1} / N_{0}=15 \mathrm{~dB}, \varepsilon_{1}=\varepsilon_{2}=0.01 \\
& r_{2}=2 r_{1}, s_{2} / s_{1}=r_{2} / r_{1} \\
\text { MAC Layer : } & q_{1}=q_{2}=1, X_{1, \min }=X_{2, \min }=0 \\
& X_{1, \max }=X_{2, \max }=10 \\
\text { CAC Layer : } & \lambda_{1}=1, \mu_{1}=\mu_{2}=1 \\
& \gamma_{1}=\gamma_{2}=0.1, \varpi_{2}=2, \varpi_{1}=1 .
\end{aligned}
$$

Fig. 10 shows the throughput comparison between the joint optimal admission policy and the complete sharing policy. By setting the class- 1 arrival rate and departure rate at $\lambda_{1}=1$ and $\mu_{1}=1$, we observe the system where the class- 2 arrival rate $\left(\lambda_{2}\right)$ varies from 1 to 100 . From the result, the throughputs are greatly improved by the optimal policy for both BPSK and QPSK. For example, at $\lambda_{2} / \lambda_{1}=10$, the throughput increases by $35 \%$. Furthermore, when $\lambda_{2} / \lambda_{1}>20$, the throughput is improved by more than $40 \%$. 


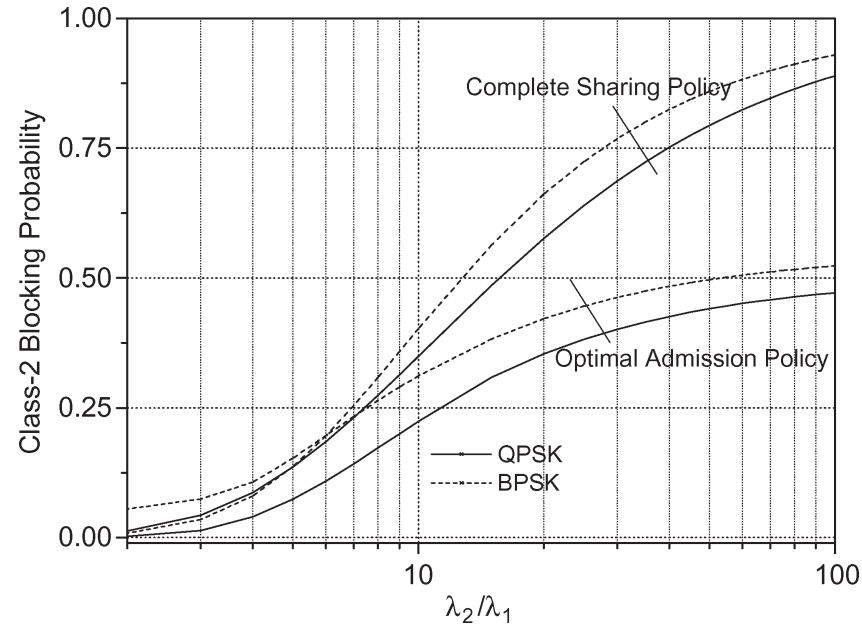

Fig. 11. Optimal admission policy versus complete sharing policy. Class2 blocking probability plotted with $G=500$, targeted SINR $=2 \mathrm{~dB}$, $E_{1} / N_{0}=15 \mathrm{~dB}, \varepsilon_{1}=\varepsilon_{2}=0.01, r_{2}=2 r_{1},\left(s_{2} / s_{1}\right) /\left(r_{2} / r_{1}\right)=1, \lambda_{1}=$ $\mu_{1}=\mu_{2}=1$, and $\gamma_{1}=0.1$.

Fig. 11 represents the class-2 blocking probability versus $\lambda_{2} / \lambda_{1}$. By fixing the class- 1 blocking probability at 0.1 , we see that the class-2 blocking probability noticeably reduces by $50 \%$, particularly when $\lambda_{2} / \lambda_{1}>20$.

As seen from the results in Figs. 10 and 11, the proposed optimal policy can improve performance by up to $50 \%$ in terms of throughput and blocking probability. In addition, this optimal policy is applicable for both BPSK- and QPSK-based VSG-CDMAs.

2) Optimal Admission for an Integrated Voice and Data System: Consider a BPSK-modulated integrated voice and data VSG-CDMA with the following parameters: $G=500$, $E_{v} / N_{0}=15 \mathrm{~dB}$, targeted SINR $\left(\zeta_{d}\right)=2 \mathrm{~dB}, \varepsilon_{d}=0.01, r_{d}=$ $2 r_{v},\left(s_{d} / s_{v}\right) /\left(r_{d} / r_{v}\right)=1$, and $x_{v}=50$. Again, assuming voice traffic is given priority over the data, then the admission policy is regarded as complete partitioning [26]. Denote $M_{v}$ and $M_{d}$ by the number of classes for voice and data, and let $M_{v}=M_{d}=1$. Under complete partitioning policy, the data is treated as a best-effort traffic. Then, the admission region of data service can be expressed as

$$
\begin{aligned}
\Psi_{d}= & \left(x_{d, 1}, \ldots, x_{d, m_{d}}\right) \in \mathcal{I}_{+}^{M}: \sum_{m=1}^{M_{d}} x_{d, m} \\
& \times\left(\frac{\ln \left[V\left(m \zeta_{d, m}\right)\right]+6 \ln \left[U\left(m \zeta_{d, m}\right)\right]}{\ln \left[\left(1-\varepsilon_{d, m}\right) V\left(m \zeta_{d, m}\right)\right]+\frac{m s_{d, 1} / s_{d, m}}{E_{1} / N_{0}} \zeta_{d, m}}\right) \\
& \left.\leq 1-\Psi_{v}\right\} \\
\Psi_{v}= & \sum_{m=1}^{M_{v}} q_{v, m} x_{v, m} \\
& \times\left(\frac{\ln \left[V\left(m \zeta_{v, m}\right)\right]+6 \ln \left[U\left(m \zeta_{v, m}\right)\right]}{\ln \left[\left(1-\varepsilon_{v, m}\right) V\left(m \zeta_{v, m}\right)\right]+\frac{m s_{v, 1} / s_{v, m}}{E_{1} / N_{0}} \zeta_{v, m}}\right)
\end{aligned}
$$

where $\Psi_{v}$ can be interpreted as the capacity occupied by voice users. As a result, the optimal cross-layer admission control is

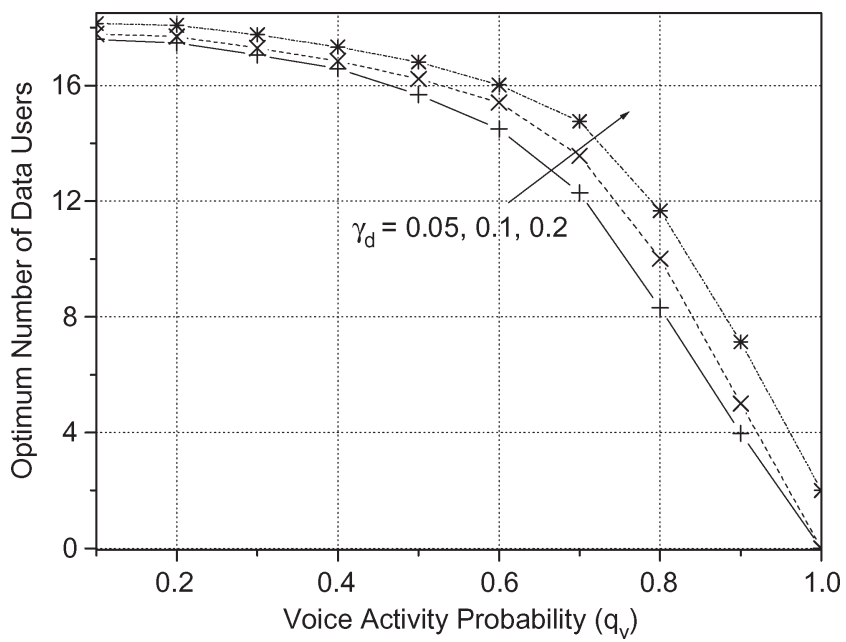

Fig. 12. Optimal admission policy. Optimum number of data users versus voice activity probability in a BPSK-based VSG-CDMA with $G=$ 500 , targeted SINR $=2 \mathrm{~dB}, E_{v} / N_{0}=15 \mathrm{~dB}, \varepsilon_{d}=0.01, r_{d}=2 r_{v}$, $\left(s_{d} / s_{v}\right) /\left(r_{d} / r_{v}\right)=1$, and $x_{v}=50$.

given by

$$
\max \sum_{\mathbf{x} \in \Psi_{d}} \sum_{\mathbf{a} \in \mathbf{A}_{\mathbf{x}}} \sum_{m \in M_{d}} \varpi_{m} x_{d, m} \tau_{\mathbf{x a}} u_{\mathbf{x a}}
$$

subject to the constraints and system-state variables in (52).

In Fig. 12, the optimum number of data users is plotted against voice activity factor $\left(q_{v}\right)$ in the case of a different blocking probability, i.e., $\gamma_{d}=0.05,0.1$, and 0.2 . The traffic intensity of the data is maintained at $\lambda_{d} / \mu_{d}=20$. With this figure, we can optimally select the number of data users for different voice load and blocking probability constraints. For example, at $q_{v}=0.5$ and $\gamma_{d}=0.1$, the optimum number of data users is 16 .

\section{CONCLUSION}

We have proposed a new linear programming approach for throughput maximization on the uplink of a multiclass VSGCDMA in Rayleigh fading in the case of both BPSK and QPSK-modulated systems. Based on the accurate interference model, exact closed-form expressions of the outage probability have been derived and used as a PHY layer constraint of the maximization problem. We have found that it is possible to transform the nonlinear constraint into a set of equivalent linear expressions. This facilitates the introduction of a new linear programming approach for the throughput maximization, which considerably reduces the computational complexity in comparison to the known nonlinear approaches. Owing to the simplicity of our linear programming approach, we can include the constraints of higher layers, i.e., MAC and CAC layers, in the proposed linear formula. This allows us to formulate a new linear MAC-PHY optimization and cross-layer (CAC-MACPHY) optimal admission policy.

In the case of the MAC-PHY optimization, the numerical results reveal that the throughput is maximized, at low background noise level, when every user (of every data rate) retains the same bit energy, particularly in the case where the difference in data rates is high. However, at high background noise, the throughput would be maximized when a larger amount of bit 
energy is allocated to the high-rate transmissions. For the joint optimal policy, the results show that the throughput and the blocking probability are improved up to $50 \%$ in comparison with the conventional admission policy, which is known as the complete sharing policy.

\section{REFERENCES}

[1] D. Ayyagari and A. Ephremides, "Optimal control in cellular DS-CDMA systems with multimedia traffic," IEEE Trans. Wireless Commun., vol. 2, no. 1, pp. 195-202, Jan. 2003.

[2] C. Li and S. Papavassiliou, "Joint throughput maximization and fair scheduling in uplink DS-CDMA systems," in Proc. IEEE Sarnoff Symp. Wired Wireless Commun., Apr. 2004, pp. 193-196.

[3] L. Jiabin, Z. Shihua, and L. Wenyuan, "Optimization of effective data rate under Rayleigh multipath fading in multimedia DS-CDMA systems," in Proc. IEEE VTC, Oct. 2003, vol. 4, pp. 2516-2520.

[4] M. K. Karakayali, R. Yates, and L. Razumov, "Throughput maximization on the downlink of a CDMA system," in Proc. IEEE WCNC, Mar. 2003, vol. 2, pp. 894-901.

[5] T. C. Wong, J. W. Mark, and K.-C. Chua, "Joint connection level, packet level, and link level resource allocation for variable bit rate multiclass services in cellular DS-CDMA networks with QoS constraints," IEEE J. Sel Areas Commun., vol. 21, no. 10, pp. 1536-1545, Dec. 2003.

[6] V. Rodriguez, D. J. Goodman, and Z. Marantz, "Power and data rate assignment for maximal weighted throughput in 3G CDMA: A global solution with two classes of users," in Proc. IEEE WCNC, Mar. 2004, vol. 4, pp. 2201-2206.

[7] S. Ulukus and L. J. Greenstein, "Throughput maximization in CDMA uplinks using adaptive spreading and power control," in Proc. IEEE 6th Int. Symp. Spread Spectrum Tech. Appl., Sep. 2000, vol. 2, pp. 565-569.

[8] S. A. Jafar and A. Goldsmith, "Adaptive multicode CDMA for uplink throughput maximization," in Proc. IEEE VTC, May 2001, vol. 1, pp. 546-550.

[9] P. Sedtheetorn and K. A. Hamdi, "Optimal cross-layer design for throughput maximization of multi-class VSG CDMA in Rayleigh fading channel," in Proc. IEEE WCNC, Apr. 2006, vol. 2, pp. 751-755.

[10] D. I. Kim, E. Hossain, and V. K. Bhargava, "Integrated rate and error control in variable spreading gain WCDMA systems," in Proc. IEEE ICC, Jun. 2001, vol. 5, pp. 1362-1366.

[11] R. Vannithamby and E. S. Sousa, "Performance of multi-rate data traffic using variable spreading gain in the reverse link under wideband CDMA," in Proc. IEEE VTC, May 2000, vol. 2, pp. 1155-1159.

[12] I. Chin-Lin and K. K. Sabnani, "Variable spreading gain CDMA with adaptive control for integrated traffic in wireless networks," in Proc. IEEE VTC, Jul. 1995, vol. 2, pp. 25-28.

[13] J. Cheng and N. C. Beaulieu, "Accurate DS-CDMA bit-error probability calculation in Rayleigh fading," IEEE Trans. Wireless Commun., vol. 1, no. 1, pp. 3-15, Jan. 2002.

[14] M. Fan, C. Hoffmann, and K.-Y. Siu, "Error-rate analysis for multirate DS-CDMA transmission schemes," IEEE Trans. Commun., vol. 51, no. 11, pp. 1897-1909, Nov. 2003.

[15] S. J. Lee, T. S. Kim, and D. K. Sung, "Bit-error probability of multicode direct-sequence spread-spectrum multiple-access systems," IEEE Trans. Commun., vol. 49, no. 1, pp. 31-34, Jan. 2001.

[16] K. A. Hamdi, "Exact probability of error of BPSK communication links subjected to asynchronous interference in Rayleigh fading environment," IEEE Trans. Commun., vol. 50, no. 10, pp. 1577-1579, Oct. 2002.

[17] M. B. Pursley, "Performance evaluation for phase-coded spread-spectrum multiple access communication-Part I: System analysis," IEEE Trans. Commun., vol. COM-25, no. 1, pp. 795-799, Aug. 1977.

[18] P. Sedtheetorn and K. A. Hamdi, "Throughput analysis of multirate VSG CDMA wireless packet communication systems in Rayleigh fading environment," in Proc. IEEE WCNC, Mar. 2005, vol. 1, pp. 158-163.

[19] J. S. Lehnert and M. B. Pursley, "Error probability for binary directsequence spread-spectrum communications with random signature sequence," IEEE Trans. Commun., vol. COM-35, no. 1, pp. 87-98, Jan. 1987.

[20] R. K. Morrow and J. S. Lehnert, "Bit-to-bit error dependence in slotted DS/SSMA packet systems with random signature sequences," IEEE Trans. Commun., vol. 37, no. 1, pp. 1052-1061, Oct. 1989.

[21] E. Geraniotis and B. G. Ghaffari, "Performance of binary and quaternary direct sequence spread-spectrum multiple-access systems with random signature sequences," IEEE Trans. Commun., vol. 39, no. 5, pp. 713-724, May 1991.
[22] T. M. Lok and J. S. Lehnert, "Error probabilities for generalized quadraphase DS/SSMA communication systems with random signature sequences," IEEE Trans. Commun., vol. 44, no. 7, pp. 876-885, Jul. 1996.

[23] G. L. Stuber, Principles of Mobile Communication, 2nd ed. Norwell, MA: Kluwer, 2002.

[24] K. S. Gilhousen, I. M. Jacobs, R. Padovani, A. J. Viterbi, L. A. Weaver, Jr. and C. E. Wheatley, "On the capacity of a cellular CDMA system," IEEE Trans. Veh. Technol. Commun., vol. 40, no. 2, pp. 303-312, May 1991.

[25] L. Kleinrock, Queueing Systems, vol. I. Hoboken, NJ: Wiley, 1975.

[26] K. W. Ross, Multiservice Loss Models for Broadband Telecommunication Networks. London, U.K.: Springer-Verlag, 1995.

[27] K. W. Ross, "Optimal circuit access policies in an ISDN environment: A Markov decision approach," IEEE Trans. Commun., vol. 37, no. 9, pp. 934-939, Sep. 1989.

[28] F. Yu, V. Krishnamurthy, and V. C. M. Leung, "Cross-layer optimal connection admission control for variable bit rate multimedia traffic in packet wireless CDMA networks," in Proc. IEEE GLOBECOM, Nov. 29, 2004 vol. 5, pp. 3347-3351.

[29] C. Comaniciu and H. V. Poor, "Jointly optimal power and admission control for delay sensitive traffic in CDMA networks with LMMSE receivers," IEEE Trans. Signal Process., vol. 51, no. 8, pp. 2031-2042, Aug. 2003.

[30] T. S. Rappaport, Wireless Communications: Principles and Practices, 2nd ed. Englewood Cliffs, NJ: Prentice-Hall, 2002.

[31] D. P. Bertsekas, Dynamic Programming and Stochastic Control, 2nd ed. Englewood Cliffs, NJ: Prentice-Hall, 2002.

[32] W. H. Tranter, K. S. Shanmugan, T. S. Rappaport, and K. L. Kosbar, Principles of Communication Systems Simulation With Wireless Applications. Englewood Cliffs, NJ: Prentice-Hall, 2004.

[33] H. C. Tijms, Stochastic Modelling and Analysis: A Computational Approach. Hoboken, NJ: Wiley, 1986.

[34] I. F. Akyildiz, D. A. Levine, and I. Joe, "A slotted CDMA protocol with BER scheduling for wireless multimedia networks," IEEE/ACM Trans. Netw., vol. 7, no. 2, pp. 146-158, Apr. 1999.

[35] W. Leinberger, G. Karypis, and V. Kumar, "Multi-capacity bin packing algorithms with application to job scheduling under multiple constraints," in Proc. IEEE Parallel Process., Sep. 1999, pp. 404-412.

[36] Y. C. Samuel, CDMA RF System Engineering. Norwood, MA: Artech House, 1998.

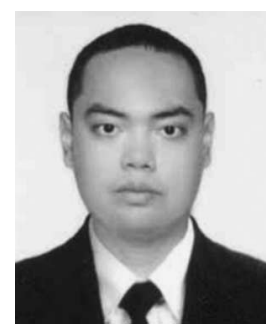

Pongsatorn Sedtheetorn (M'07) received the Bachelor and Master degrees in electrical engineering from Chulalongkorn University, Bangkok, Thailand, in 1998 and 2001, respectively, and the Ph.D. degree from the University of Manchester, Manchester, U.K., in 2007.

$\mathrm{He}$ is currently a Lecturer with the Department of Electrical and Computer Engineering, Mahidol University, Nakhon Pathom, Thailand. His research interests include spread-spectrum communications, radio wave channel modeling, frequency planning for cellular systems, media access control protocol and admission control protocol for wireless communications, and packet radio communication systems.

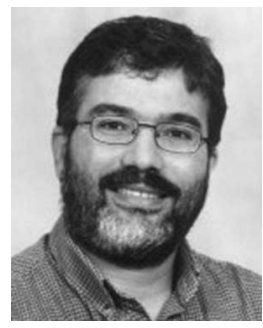

Khairi Ashour Hamdi (SM'02) received the B.Sc. degree in electrical engineering from the Alfateh University, Tripoli, Libya, in 1981, the M.Sc. degree (with Distinction) from the Technical University of Budapest, Budapest, Hungary, in 1988, and the Ph.D. degree from the Hungarian Academy of Sciences, Budapest, in 1993.

He was with the Department of Electronic Systems Engineering, University of Essex, Colchester, U.K. He is currently with the School of Electrical and Electronic Engineering, University of Manchester, Manchester, U.K. His research interests include performance analysis of communication systems, wireless ad hoc networks, interference and coexistence in the license-exempt frequency bands, and spread-spectrum communication and networks.

Dr. Hamdi was a British Telecom Research Fellow in 2002. 